\title{
Social and Behavioral Consequences of the Vietnam Experience among American Legionnaires ${ }^{1}$
}

\author{
Jeanne Mager Stellman, * Steven D. Stellman, $\dagger$,2 and \\ JOHN F. SOMMER, JR. \\ *School of Public Health, Columbia University; +Department of Epidemiology and Statistics, \\ American Cancer Society, New York, New York; and $\$$ National Veterans Affairs and \\ Rehabilitation Commission, The American Legion, Washington, D.C.
}

Received November 2, 1987

\begin{abstract}
One aspect of a cross-sectional questionnaire study of a sample of Vietnam veterans belonging to The American Legion was devoted to analysis of social and behavioral consequences of service in Southeast Asia. Members of the study population were stratified by whether they served in Southeast Asia and, within the Southeast Asia group, by varying degrees of exposure to combat and to herbicides. Using validated scales for behavioral affect and for the exposure measures for combat and herbicides, a wide range of adverse effects was observed. Men who faced high levels of combat intensity were found to be at greater risk for divorce and for generally being less happy and satisfied with their lives, their marriages, their role as fathers, and as sexual human beings. Although in this population they have reached comparatively high levels of education, they earn significantly less money than peers of their same age and educational attainment. Vietnam veterans returning from combat were found to exhibit higher levels of behavioral disturbances, with mean scores for depression, anxiety, irritation, feelings of helplessness, and physical signs of depression significantly worse than noncombat peers. The lifestyle of combat veterans also continues to place them at greater risk for poor health. They smoke, drink, and use prescription drugs at rates significantly greater than the other veterans in this study. Combat veterans have had a significantly poorer rate of reduction and cessation of smoking and drinking than others in this population. Evidence is also presented which shows that for some of the behavioral outcomes measured, a negative interactive effect of concurrent exposure to herbicides is present. 1988 Academic Press, Inc.
\end{abstract}

\section{INTRODUCTION}

The social and psychological readjustment of Vietnam veterans has been an area of public debate and a potent political issue for many years. In 1977 the G.I. Bill Improvement Act (Public Law 95-202) mandated that the Veterans Administration undertake a national independent study of veteran adjustment to determine the extent to which readjustment problems were a real and persistent issue. This mandate resulted in a massive study "Legacies of Vietnam: Comparative Adjustment of Veterans and Their Peers," ("Legacies") conducted by the Center for Policy Research (Egendorf et al., 1981). "Legacies" showed that men who ex-

\footnotetext{
${ }^{1}$ Address reprint requests to Foundation for Worker, Veteran and Environmental Health, Inc., 117 St. Johns Place, Brooklyn, NY 11217.

${ }^{2}$ Present address: New York City Department of Health, New York, NY 10013.
} 
perienced service in Vietnam suffered from significantly more social adjustment problems than their peers with a different, noncombat service experience.

There appears to be growing consensus in the research community that returning veterans do have problems, but there is serious disagreement as to the exact nature of the problems, their intensity, and duration. Some see the problems as short-lived for the majority of veterans. They have proposed that the consequences of exposures to combat diminish and eventually disappear with time. They further attribute any long-lasting social and behavioral effects among veterans to defects and deficiencies that the veterans brought with them into the armed services (Worthington, 1977, 1978).

A research data base has been developing over the past several years, from clinical observations (e.g., Figley, 1978) and from studies such as "Legacies," which lends credence to a residual stress theory. "Legacies" showed that exposure to combat bears a direct and significant relation to current alcohol and drug use, arrests, medical problems, and stress-related symptoms. Among white veterans, excessive drinking was a problem, while among the black veterans studied excess use of drugs was observed ("Legacies": Volume III). The effects were primarily observed in men who served in the armed forces during 1968 or later. Educational and work careers of these combat veterans were also affected. Vietnam veterans with combat exposures were found to hold lower level jobs than veterans who served outside the war zone ("Legacies": Volume II).

One finding of particular consequence to the work described here is that, for many, service in Vietnam alone was not the determining factor of the adverse social and behavioral consequences observed. Rather the intensity of combat to which the veteran was exposed was predictive of these outcomes.

There has also been scientific argument and public debate about whether the apparent effects of acute exposure to the life-threatening conditions of combat are being observed because a preponderance of those who served were less socially and psychologically fit and, hence, more susceptible to readjustment problems after the service. A recent prospective study of Vietnam veterans, with and without combat experience, and of men who did not serve at all, addresses this issue. The population from which the 1500-man Vietnam Era sample was drawn had been assembled as a cohort of ninth graders, in a nationwide study, in 1960 . That survey collected comprehensive information on academic abilities, interests, personalities, home life, and plans for future schooling, work, and military service (Card, 1980). The data substantiate the claim that, on the whole, the men who served in Vietnam were comparable to their peers who did not. Card also found that nonveterans were ahead of veterans in income and job prestige. She observed that Vietnam veterans suffered from greater levels of psychological distress than nonveterans, in proportion to the intensity of combat which they experienced. Card did not observe that alcohol consumption increased with increasing combat exposure. This is in disagreement with the work of Laufer et al. ("Legacies": Vol. III) and with our own data presented below. Card also did not observe adverse effects of military service on overall marital stability, but did observe a decrease in general satisfaction with life.

Given this background of persistent evidence of readjustment problems, the 
American Legion study of Vietnam veterans sought to answer the following questions on the social and behavioral consequences of service during the Vietnam War. Did men who served in heavy combat areas suffer long-term economic consequences, such as lower family income, compared to men in other zones and men who did not serve in Southeast Asia? Has there been an adverse effect of combat on the marital and parental lives of Vietnam veterans? Are men who experienced combat in general less happy and less satisfied with their lives than men who did not fight or did not serve in Southeast Asia? Has service in Vietnam affected the health behaviors of veterans by influencing their smoking, drinking, and drug usage patterns? Did service in Southeast Asia in general, and in a combat zone in particular, result in an increase in affective disorders, such as depression and anxiety, compared to service elsewhere?

A second line of investigation concerned the possible role of exposure to herbicides such as Agent Orange in the social and behavioral outcomes under study. Our conceptualization for stress in veterans is similar to the model in which both the physical and psychosocial environment predict the health and well-being of workers in various settings (House et al., 1979; Stellman and Snow, 1986). Following this model, for each of the outcomes under study we asked whether exposure to herbicides during service in Southeast Asia exacerbates the adverse effects of combat or possibly exerts an independent effect. [Post-traumatic stress disorder, another important psychosocial outcome, was also explored in this study and is the subject of the fourth paper in this series (Snow et al., 1988).]

\section{METHODS AND MATERIALS}

The study has a cross-sectional design in which various outcome measures are compared between groups of men who served in Southeast Asia, stratified by the combat intensity they experienced, and men who served elsewhere during the same period. The men were randomly selected from the membership rosters as of October 15, 1983, in six Departments of The American Legion encompassing the states of Colorado, Indiana, Maryland, Minnesota, Ohio, and Pennsylvania. The random sampling procedures and details of the selection process are given in a preceding paper (Stellman, et al., 1988a).

The survey consisted of a self-administered questionnaire requiring between 45 $\min$ and $1 \mathrm{hr}$ for completion. Several sections of the questionnaire were designed to measure social and behavioral outcomes of interest. These are given in Table 1. Where an outcome is measured by a scale, the items in the scale and its reliability, as measured by the Cronbach $\alpha$, are given. Only scales achieving a reliability of $\alpha>0.67$ were utilized.

An attempt was made to use items and measures that were compatible with the work of other researchers and with our own previous research. Several of the measures of behavioral affect are derived from the Psychiatric Epidemiology Research Interview (PERI) and other well-known sources (Dohrenwend et al., 1980; Quinn and Shepard, 1974; Caplan et al., 1975). Information on intensity, duration, and changes in smoking, drinking, and drug usage was elicited. Demographic characteristics, such as age, race, income, and education, were also obtained. 
TABLE 1

Measures Used in the Analysis of Social and Behavioral Outcomes

Social adjustment

Marital well-being $(\alpha=0.78)$

(Scored $1=$ Very often, $2=$ Fairly often, $3=$ Sometimes, $4=$ Almost never, $5=$ Never):

"During the past six months how often have you experienced . . . about person you live with?"

_ feeling uncomfortable

— thinking person doesn't really love you

_ t thinking you don't really love person

_ losing temper

__ physically striking

_ feeling affection (scored $5=$ Very often $\ldots 1=$ Never)

Satisfaction with parenting $(\alpha=0.87)$

(Scored $1=$ Very dissatisfied, $2=$ Somewhat dissatisfied, $3=$ Somewhat satisfied, $4=$ Very satisfied):

"During the past six months, how satisfied were you with . . ."

kind of parent you've been

— how well you understand your children

— getting along with your children

_ time spent with your children

— way your children are turning out

— respect your children show

Sexual satisfaction $(\alpha=0.77)$

(Scored 1 = Very often, $2=$ Fairly often, $3=$ Sometimes, $4=$ Almost never, $5=$ Never $)$ :

"How often have you ...?" had no interest in sex

— found yourself getting no pleasure from sex

_ had trouble reaching a climax

Single-item measures

(Scored 1 = Very dissatisfied, $2=$ Somewhat dissatisfied, $3=$ Somewhat satisfied, $4=$ Very satisfied):

"During the past six months, how satisfied ...."

Mate's satisfaction with relationship

_ has your mate been with the relationship?

Satisfaction with mate relationship - were you with the relationship you have had with the person you live with?

(Scored $1=$ Not at all, $2=$ Slightly, $3=$ Moderately, $4=$ Very):

Happiness

Taking all things together, how happy are you these days?

Life satisfaction

In general, how satisfying is your life? 
TABLE 1-Continued

\section{Affective state}

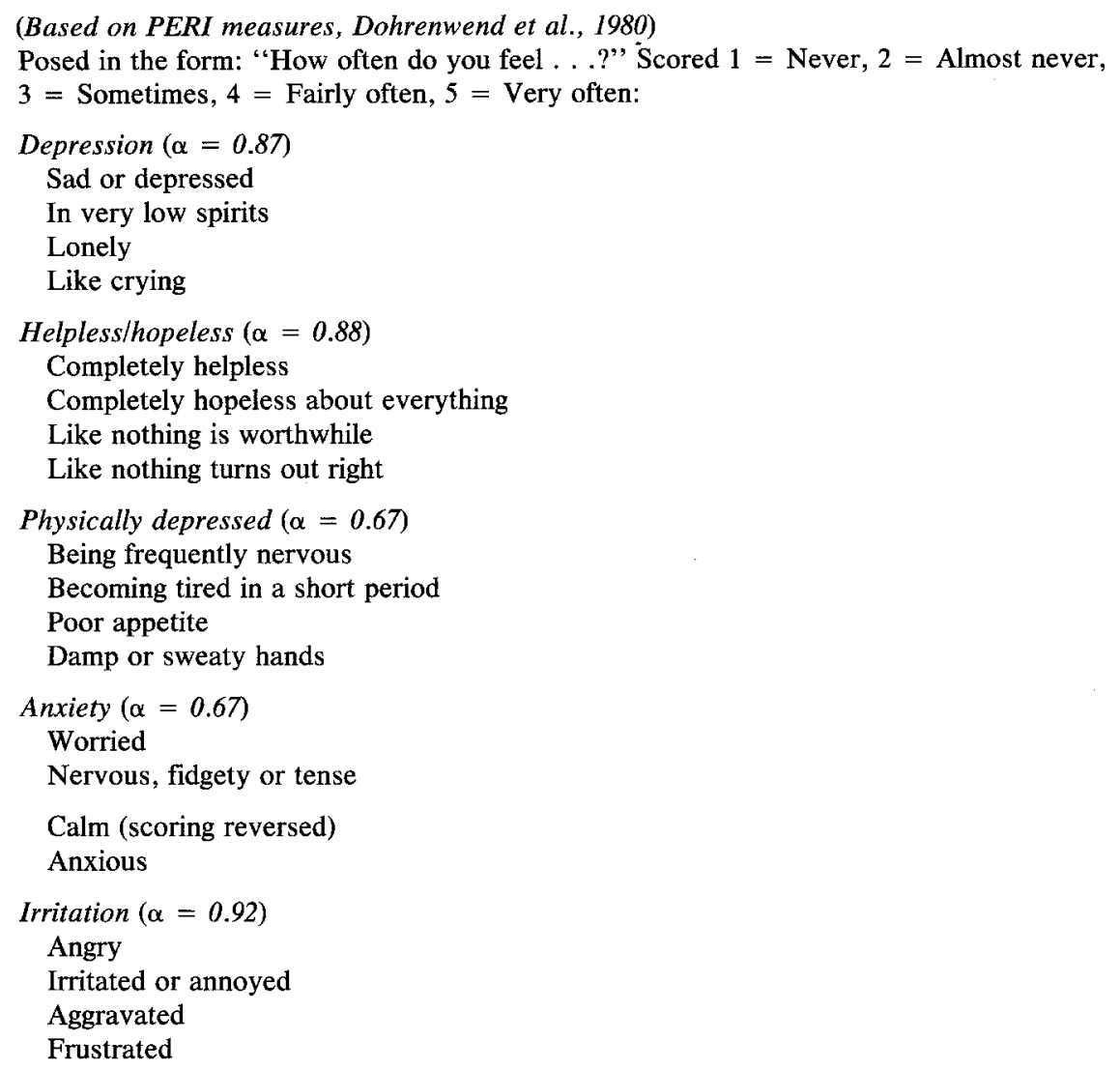

The two major independent variables of interest were combat and exposure to herbicides. Combat intensity was measured by a highly reliable $(\alpha=0.96)$ eightitem scale used by several other researchers (Figley, 1978; Frye and Stockton, 1982; Roberts et al., 1982). Further details about this scale and its distribution among the Legionnaires surveyed were given in the first of this series (Stellman $e t$ al., 1988a).

An individual's score on the combat scale could range between 8 and 40. Subjects were classified as experiencing low (8-15), medium (16-25), or high (26-40) scores. The percentages of Vietnam veterans so categorized were 42.5, 38.1, and $19.4 \%$, respectively. Classification as to herbicide exposure was based on a numerical index developed by the authors, which rated each veteran's closeness in time and space to known military missions in which herbicides were sprayed or dropped. The dates and locations of service in Vietnam were elicited in the questionnaire by asking respondents who served in Southeast Asia to fill out a location matrix developed earlier by us (Stellman and Stellman, 1986). 


\section{RESULTS}

\section{Social and Demographic Characteristics of the Sample}

Age and race. Ninety-six percent of the 6810 respondents were born after 1930 , and $71.4 \%$ were born during the decade 1940-1949, making the cohort highly homogeneous with respect to age. Of the 6810 subjects, $2858(42.0 \%)$ had served in Southeast Asia, and 3933 had not. Service location could not be determined for 19 subjects.

The racial composition of the study population is $98.5 \%$ white.

Education and income. The degree of educational attainment of Southeast Asia veterans is compared to that of men who served elsewhere in Table 2. The great majority of subjects have completed high school or technical school. Almost 13\% have completed college, and an additional $5 \%$ have had professional or graduate education. As would be expected of a cross-sectional sample of men in early middle age, incomes are slightly higher among the older men. As can be seen from Table 2, when Southeast Asia veterans are compared to all others, there is little difference in educational attainment. There were no significant income differences between Southeast Asia veterans and non-Southeast Asia subjects, except for the relatively small cohort born between 1930-1939, where the income of Southeast Asia veterans was slightly higher (by about $\$ 800$ per year).

A different picture emerges, however, when income and education are analyzed in relation to combat experience. Figure 1 shows the mean family income level according to level of combat. There was a significant $(P<0.001)$ trend toward lower family incomes with increasing combat levels. This trend held true within individual birth cohorts. In every cohort born after 1930, the mean family income was greatest in the low-combat group. (Among subjects born before 1930, there was a small difference in favor of high combat; we cannot be certain whether this group of careerists experienced combat in Vietnam, in earlier wars, or both.)

Since it is well known that education is predictive of income, we studied the effect of both combat and educational attainment on income by carrying out a multiple regression analysis where combat and education were entered as independent variables, along with an interaction term, education $\times$ combat. Only men

TABLE 2

Educational Attainment by Location of Service

\begin{tabular}{|c|c|c|}
\hline Educational level & $\begin{array}{c}\text { Served in SEA } \\
\quad N=2839\end{array}$ & $\begin{array}{l}\text { Served elsewhere } \\
\qquad N=3878\end{array}$ \\
\hline & \multicolumn{2}{|c|}{ Percentage } \\
\hline Less than high school & 6.3 & 5.5 \\
\hline High school & 37.3 & 40.8 \\
\hline Some college & 22.9 & 22.2 \\
\hline Vocational/technical school & 16.1 & 15.0 \\
\hline College graduate & 13.0 & 12.3 \\
\hline Graduate/professional school & 4.4 & 4.3 \\
\hline
\end{tabular}




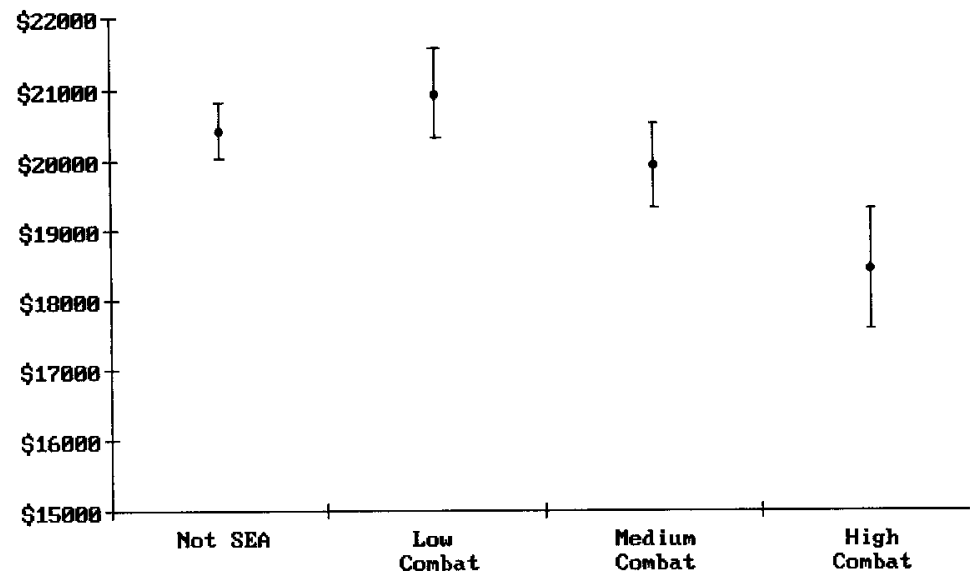

Fig. 1. Mean income level in dollars by level of combat. Bars indicate upper and lower $95 \%$ confidence limits.

who served in Southeast Asia were considered in the regression. As expected, education was a significant predictor of income, as was combat $(P<0.001)$; the interaction term, however, was not significant. The values of $\beta$ (coefficient divided by its standard error) were 0.206 for education and -0.103 for combat.

Approximate dollar differences in mean family income are shown for each birth cohort in Table 3. On the average, family income in low-combat veterans exceeded that for high-combat veterans by $\$ 2470$ per year $(P<0.01)$. Income differences were significant in four of the six birth cohorts and ranged from $\$ 480$ per year for men born $1948-1949$ to $\$ 5190$ per year $(P<0.01)$ for men born 1930-1939.

These findings demonstrate that significant family income loss has, on the average, occurred among Vietnam combat veterans, irrespective of their degree of educational attainment. It also shows the necessity of differentiating among men who served in Southeast Asia on the basis of actual wartime experience, since in

TABLE 3

Approximate Difference in Average Income Between Low- and Hrgh-Combat Southeast asia Veterans

\begin{tabular}{lc}
\hline Year of birth & Income difference (dollars) \\
\hline All years & $\$ 2470^{* *}$ \\
$1930-1939$ & $5190^{* *}$ \\
$1940-1943$ & 1750 \\
$1944-1945$ & $3560^{*}$ \\
$1946-1947$ & $3220^{* *}$ \\
$1948-1949$ & 480 \\
1950 or later & $3960^{*}$ \\
\hline
\end{tabular}

${ }^{*} P<0.05 ; * * P<0.01$. 
this instance it is exposure to high intensity combat, not merely service in Southeast Asia, that is the predictive variable.

\section{Family and Marital Relationships}

We approached this assessment of the impact of the Vietnam War on veterans with respect to their marital and family relationships in several ways. First we considered the rate of marriage and divorce or separation among the comparison groups. We also tried to assess the quality of those relationships through the use of measures of marital, parental, and sexual satisfaction, as defined in Table 1. Table 4 shows numbers of men who have been "ever married," "ever separated or divorced," and "currently separated or divorced," by location of service and level of combat intensity.

The percentage of ever-separated/divorced men was significantly higher among Southeast Asia veterans than among others $(36.7 \%$ vs $28.4 \%, P<0.001)$. The percentages rose dramatically with increasing levels of combat: low $=28.5 \%$, medium $=34.6 \%$, high $=60.4 \%$, with high-combat veterans nearly four times as likely to have experienced a separation or divorce than non-Southeast Asia veterans. Similar trends were observed for current separation or divorce: the rate among all Southeast Asia veterans was significantly higher than among others (16.1\% vs $11.0 \%, P<0.001$ ), and rose concurrently with increased levels of combat exposure. The odds in favor of current separation or divorce were nearly 3:1 in high-combat veterans relative to non-Southeast Asia subjects.

In addition to assessing the stability of marital relationships among the Legionnaires, we also attempted to assess the quality of the relationships through a scale we call marital well-being and through two single-item measures querying how satisfied the respondent was with his partner and his perception of his mate's satisfaction. These data are plotted as a function of service location and combat intensity in Figs. 2a, 2b, and $2 \mathrm{c}$ and the questions are given in Table 1. Significant

TABLE 4

Marital Status by Location of Service and Combat Intensity Level

\begin{tabular}{|c|c|c|c|c|c|}
\hline & \multirow{2}{*}{$\begin{array}{c}\text { Not in } \\
\text { Southeast } \\
\text { Asia }\end{array}$} & \multicolumn{3}{|c|}{ Combat intensity level } & \multirow{2}{*}{$\begin{array}{c}\text { All } \\
\text { SEA }\end{array}$} \\
\hline & & Low & Medium & High & \\
\hline & \multicolumn{5}{|c|}{ Numbers } \\
\hline A: Ever separated or divorced & 768 & 248 & 274 & 197 & 719 \\
\hline B: Currently separated or divorced & 297 & 114 & 117 & 84 & 315 \\
\hline \multirow[t]{2}{*}{ C: Ever married } & 2708 & 871 & 761 & 326 & 1958 \\
\hline & \multicolumn{5}{|c|}{$\begin{array}{c}\text { Separation or divorce rates } \\
\text { (percentages) }\end{array}$} \\
\hline $\mathrm{A} / \mathrm{C}$ & 28.4 & 28.5 & 34.6 & 60.4 & 36.7 \\
\hline Odds ratios ${ }^{a}$ & 1.00 & 1.01 & $1.42^{* * *}$ & $3.86^{* * *}$ & $1.47^{* * *}$ \\
\hline $\mathrm{B} / \mathrm{C}$ & 11.0 & 13.1 & 15.4 & 25.8 & 16.1 \\
\hline Odds ratios ${ }^{a}$ & 1.00 & 1.22 & $1.48^{* * *}$ & $2.82 * * *$ & $1.56 * * *$ \\
\hline
\end{tabular}

${ }^{a}$ Odds ratio relative to not in Southeast Asia $=1.00$.

*** $P<0.001$. 
a

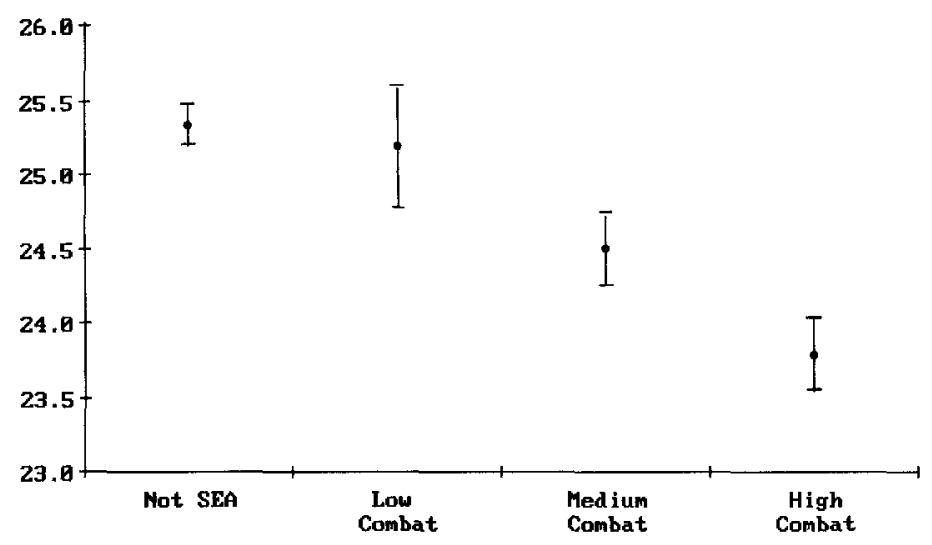

b

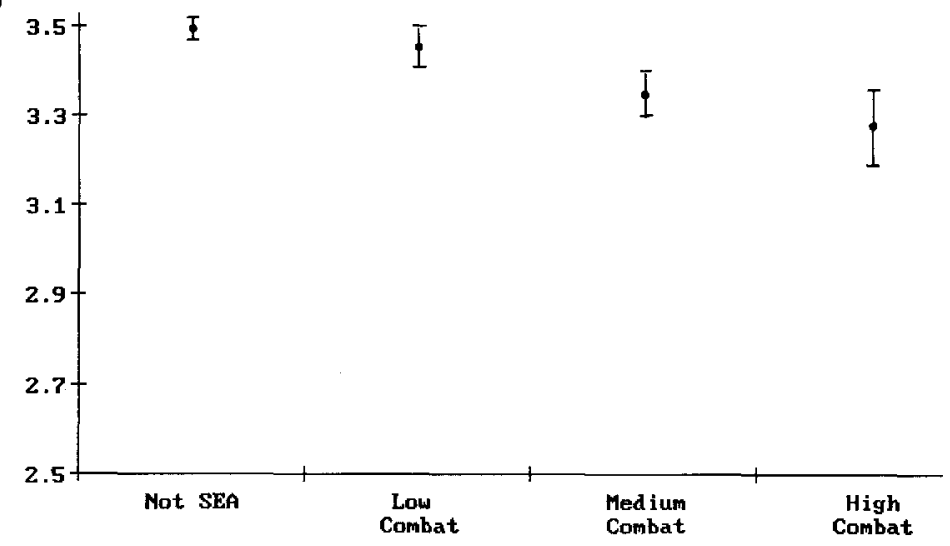

c

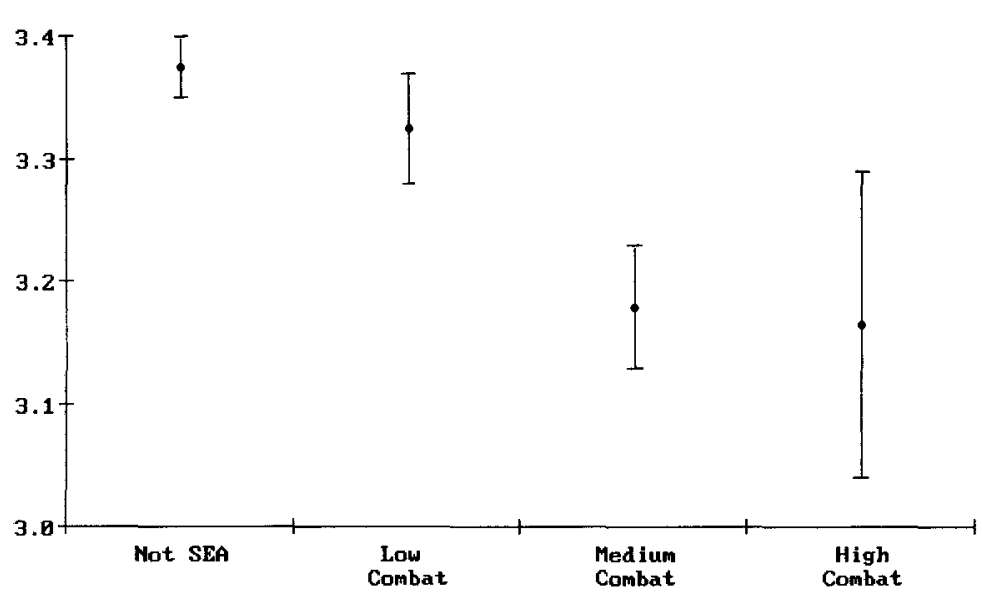

FIG. 2. (a) Mean marital well-being scale, according to level of combat. Lower score indicates increased stress. (b) Mean level of satisfaction with marital relationship. Higher score indicates increased satisfaction. (c) Mean level of satisfaction of marital partner. Higher score indicates increased satisfaction. Bars indicate upper and lower $95 \%$ confidence limits. 
differences were observed between men who experienced heavy combat compared to men who experienced low combat or who were not stationed in Southeast Asia. Differences between low combat and service elsewhere were not statistically significant and there was overlap, as well, between the medium and the heavy combat categories.

We used a scale, called sexual satisfaction (Table 1), to measure the respondents' satisfaction with their sexual relationship and the level of difficulty which they may be encountering in their sexual performance. Its mean value as a function of location of service and combat intensity is plotted in Fig. 3. The results obtained here are similar to those obtained for the measures of marital satisfaction: heavy combat significantly and negatively affects sexual satisfaction, as does exposure to medium levels of combat, but to a lesser extent. The effect of low combat intensity exposure is indistinguishable from the effect of service someplace other than Southeast Asia.

We also evaluated the degree of satisfaction that respondents had about their parenting roles using the satisfaction with parenting scale defined in Table 1 . The results are given in Fig. 4 and, as with the marital well-being and sexual satisfaction scales, a significant and consistent negative effect of medium and heavy combat compared to low combat or not being stationed in Southeast Asia was observed.

It has previously been suggested that libidinal depression is a possible consequence of exposure to dioxins or Agent Orange (Poland et al., 1971; Bogen, 1979; Suskind and Hertzberg, 1984). A multiple regression analysis approach was used to evaluate the possible effect of exposure to Agent Orange on the sexual satisfaction outcome measure. Both combat and Agent Orange exposure (as the numerical index, OE3) were entered into the equation, along with an interaction term, combat $\times$ OE3. Combat and the cross-product term were both significant predictors of this scale $(P<0.05)$, but not the Agent Orange term itself. The magnitude of the cross-product $\beta$ was approximately one-third that of combat. This result, where herbicide is not an independent predictor but only enters the

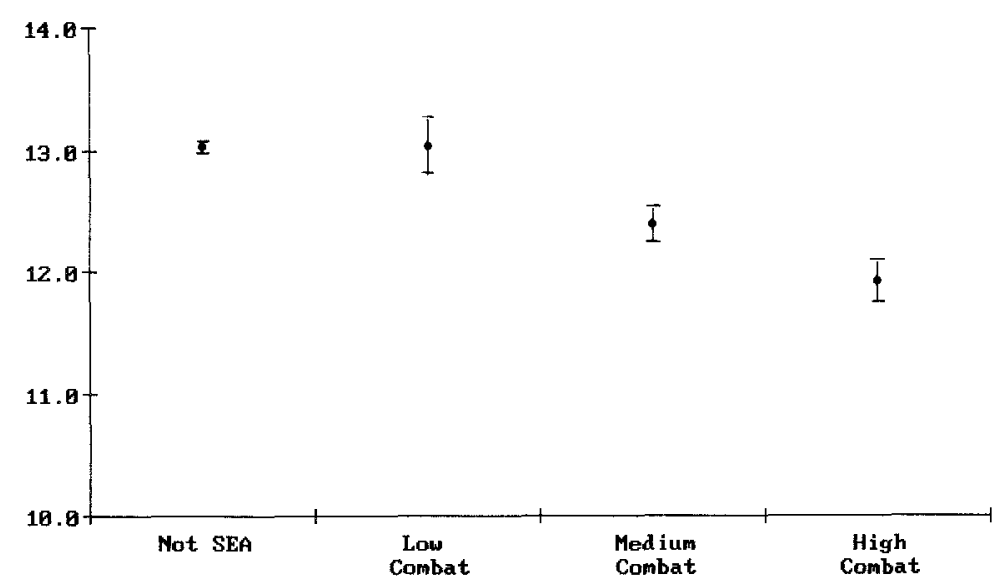

FIG. 3. Mean value of sexual satisfaction scale according to level of combat. Higher score indicates increased satisfaction. Bars indicate upper and lower $95 \%$ confidence limits. 


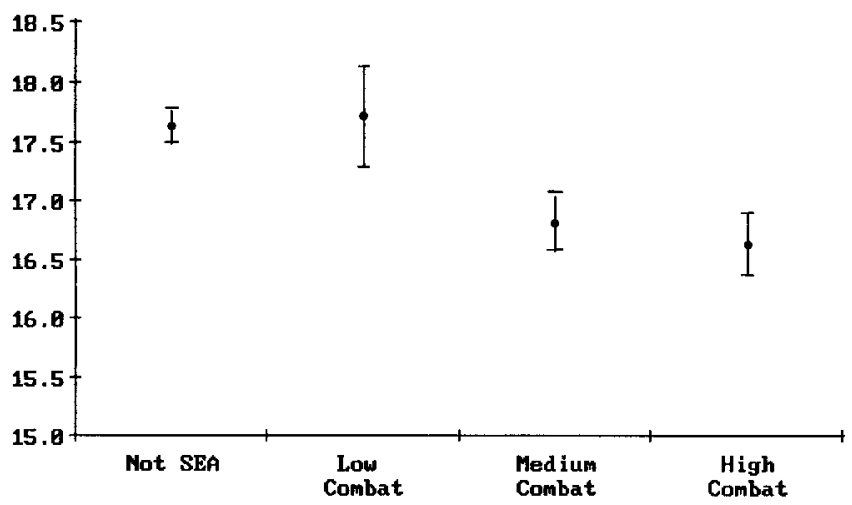

FIG. 4. Mean value of satisfaction with parenting scale according to level of combat. Bars indicate upper and lower $95 \%$ confidence limits.

equation in an interactive term, suggests that a herbicide effect which is manifest only at very high levels of combat is present. In similar regression analyses of the parental and marital outcome measures, combat alone proved significant, but not Agent Orange nor the interaction term.

\section{Measures of Life Satisfaction and Psychological Well-being}

Life satisfaction. We asked two straightforward questions about how happy people were at the time of the survey and how satisfied they were with their lives. Men in medium and heavy combat are significantly less happy and less satisfied than their low-combat or non-Southeast Asia peers, as shown in Figs. 5a and 5b.

Psychological well-being. Five measures of psychological well-being were depression, anxiety, helplessness/hopelessness, degree of anger/irritation, as well as physical symptoms of depression. We found these measures to be correlated with each other, as shown in Table 5. The correlation coefficients range from 0.42 for irritation with physical depression, to 0.72 for the correlation between helplessness and depression. They are all correlated with combat, as well, though the coefficients are smaller.

The mean score for each of these measures was significantly higher $(P<0.001)$ among men who served in Southeast Asia than among those who served elsewhere during the Vietnam Era, as shown in Table 6. In addition, the percentage of men who served in Southeast Asia and who had scores above the median for these five psychological status measures rose with the level of combat experienced. For instance, the measure of depression was above the median for $47.6 \%$ of the low-combat group, compared to $56.9 \%$ of the middle-combat group, and $66.6 \%$ of the high-combat group. Similar trends were observed for the four other scales. All trends were statistically significant $(P<0.001)$ as measured by the Mantel-extension $\chi$. Figure 6 shows the variation of the mean levels for each of the measures at different levels of combat among those who served in Southeast Asia and among a comparison group of those who served elsewhere. The graphs in Fig. 6 demonstrate a decided dose-response for each of the five measures.

The main hypothesis was that affective state, as measured by the five scales 
a

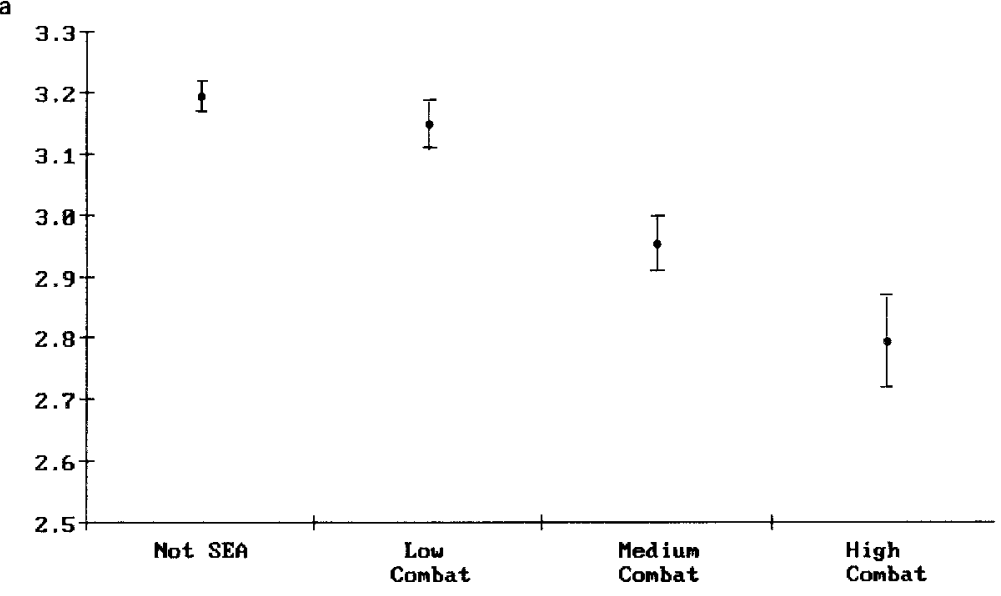

b

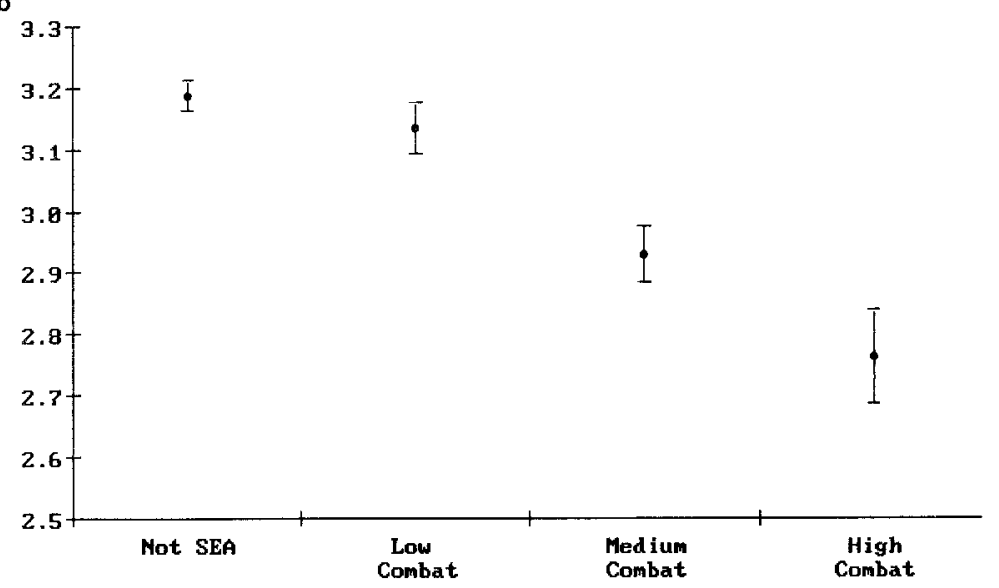

FIG. 5. Mean value of scales measuring (a) happiness and (b) satisfaction with life, according to level of combat. Bars indicate upper and lower $95 \%$ confidence limits.

used here, would be dependent upon exposure to combat in Southeast Asia. A second hypothesis dealt with the potential effects, either independent or interactive, of exposure to herbicides. We observed a superficial association between several of the scales and exposure to herbicides, as measured by the numerical index OE3 described above. As shown in Table 5, the scales are correlated with both of these independent variables. The correlation is comparatively strong with combat exposure (e.g., "helpless", $r=0.18$ and "physical depression", $r=$ 0.30) among the group with service in Southeast Asia. There is only a weak association with exposure to herbicides. All correlation coefficients were statistically significant $(P<0.001)$.

Using a median split analysis, we obtained the percentage distribution of exposure to both combat and Agent Orange (three levels each) in subjects who scored "high" (above the median) for each scale, compared with subjects who scored "low" (below the median). Only two of the five scales, those measuring 
TABLE 5

Correlation Coefficients of Psychological Well-being Scales with Each Other, and With INDEXes of Level of Combat and Agent Orange ExPosure

\begin{tabular}{|c|c|c|c|c|c|c|c|}
\hline & Depress & Anxiety & Hpless & Irritn & Physdpr & Combat $^{a}$ & Agent Orange $^{a}$ \\
\hline Depress & 1.00 & 0.69 & 0.72 & 0.65 & 0.51 & 0.22 & 0.08 \\
\hline Anxiety & & 1.00 & 0.62 & 0.64 & 0.53 & 0.22 & 0.11 \\
\hline Hpless & & & 1.00 & 0.63 & 0.48 & 0.18 & 0.08 \\
\hline Irritn & & & & 1.00 & 0.42 & 0.19 & 0.09 \\
\hline Physdprs & & & & & 1.00 & 0.30 & 0.15 \\
\hline Combat $^{a}$ & & & & & & 1.00 & 0.24 \\
\hline Agent Orange $^{a}$ & & & & & & & 1.00 \\
\hline
\end{tabular}

a Applies to Southeast Asia veterans only.

anxiety and physical depression, were related to Agent Orange exposure. After controlling for combat, the trend in odds ratios for physical depression was not statistically significant, and that for anxiety, while significant, was numerically small (odds ratio for medium combat $=1.1$; for high combat $=1.3$ ). Odds ratios of this magnitude might well be attributable to residual confounding by combat, or to chance. The association might also be partly attributable to the correlation between physical depression and physical symptoms found to be related to herbicide exposure and described in another paper in this series.

The relationship between the five well-being scales and combat exposure and Agent Orange exposure was further explored by treating each outcome measure as a continuous variable in a multiple regression analysis. Combat was a consistent significant predictor of each of the five scales. However, herbicide exposure was not, nor did analysis of covariance of any of the five scales yield significant main effects for herbicide exposure, when combat was the covariate.

TABLE 6

Means $(X)$ and Standard Errors (SE) for Five Scales Measuring Psychological Well-being for SubJects with Service in Southeast Asia Compared with Controls

\begin{tabular}{|c|c|c|c|c|}
\hline \multirow[b]{2}{*}{ Scale } & \multicolumn{3}{|c|}{ Served in Southeast Asia } & \multirow[b]{2}{*}{$P(t$ test $)$} \\
\hline & & Yes & No & \\
\hline \multirow{3}{*}{ Depressn } & $X$ & 9.30 & 8.45 & \multirow{3}{*}{$<0.001$} \\
\hline & $S E$ & 0.06 & 0.05 & \\
\hline & $\mathrm{N}$ & 2797 & 3832 & \\
\hline \multirow[t]{3}{*}{ Anxiety } & $X$ & 10.74 & 9.92 & \multirow[t]{3}{*}{$<0.001$} \\
\hline & $\mathrm{SE}$ & 0.06 & 0.05 & \\
\hline & $\mathbf{N}$ & 2692 & 3688 & \\
\hline \multirow[t]{3}{*}{ Hpless } & $X$ & 7.23 & 6.65 & \multirow[t]{3}{*}{$<0.001$} \\
\hline & $\mathrm{SE}$ & 0.06 & 0.05 & \\
\hline & $\mathrm{N}$ & 2780 & 3777 & \\
\hline \multirow[t]{3}{*}{ Irritn } & $X$ & 10.94 & 10.07 & \multirow[t]{3}{*}{$<0.001$} \\
\hline & SE & 0.07 & 0.05 & \\
\hline & $\mathbf{N}$ & 2765 & 3770 & \\
\hline \multirow[t]{3}{*}{ Physdprs } & $X$ & 5.21 & 4.69 & \multirow[t]{3}{*}{$<0.001$} \\
\hline & SE & 0.04 & 0.02 & \\
\hline & $\mathrm{N}$ & 2460 & 3460 & \\
\hline
\end{tabular}



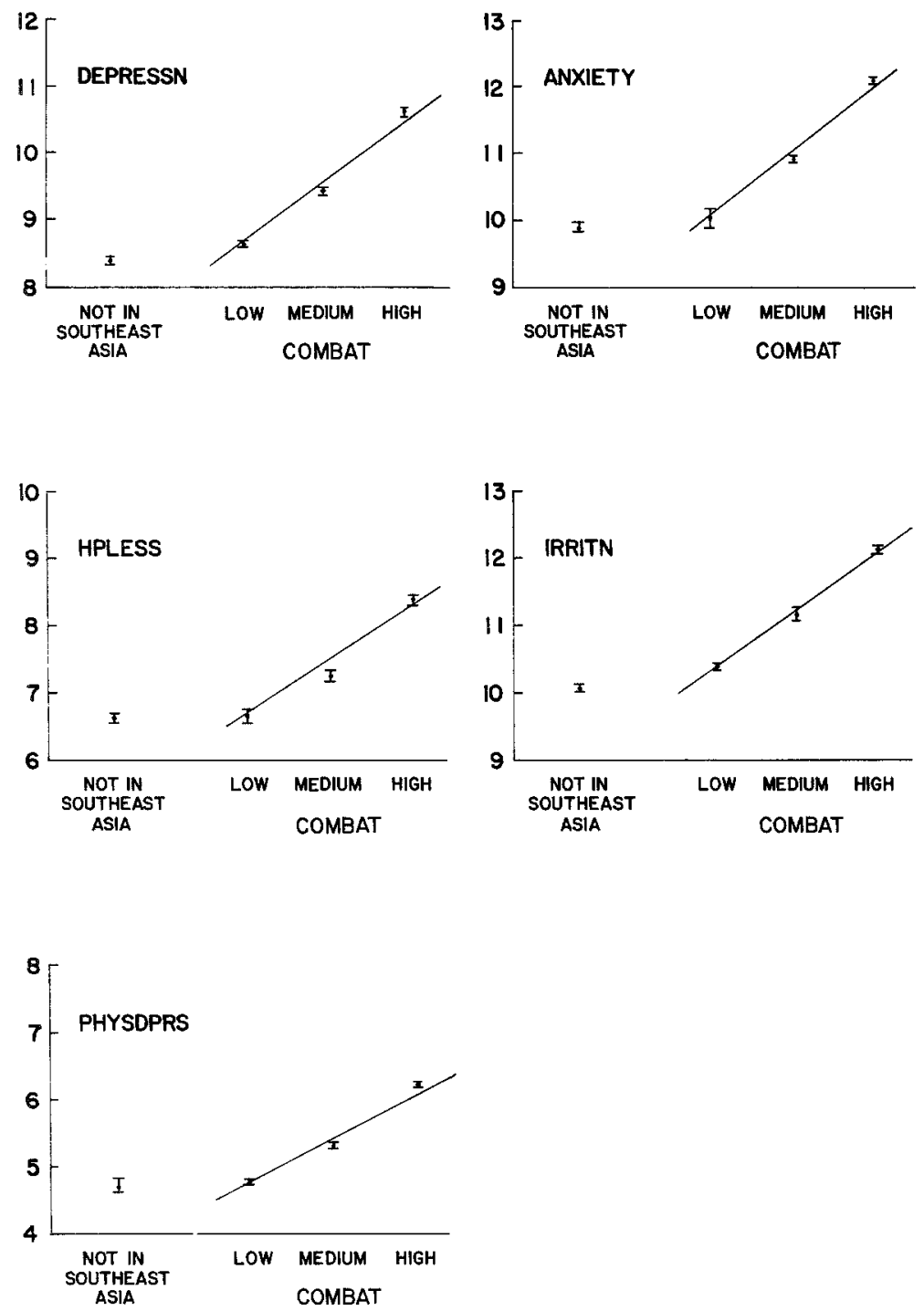

FIG. 6. Mean value of PERI scales (affective state), according to categorical level of combat (low, medium, high, not in Southeast Asia). Bars indicate upper and lower 95\% confidence limits. Superimposed on the three Southeast Asia data points is a separately computed regression line which relates each given PERI scale to combat as a continuous variable. See Table 1 for description of scales and their components.

\section{Smoking, Drinking, and Drugs}

We investigated the effect of military service on three personal habits: smoking, drinking, and use of drugs. These habits are not only indicators of social stress, but are predictors of long-term health outcomes.

For over $92 \%$ of study subjects who smoked, cigarette smoking began prior to military service; present smokers had smoked regularly an average of 2 years 
before induction or enlistment. Although military service did not "create". smokers, it had a major influence on subsequent smoking habits for many veterans.

The distribution of smoking habits according to military service, and according to level of combat in Southeast Asia, is shown in Fig. 7. As with the other outcomes analyzed here, the proportion of never-smokers was approximately the same in the Southeast Asia and non-Southeast Asia groups (21.9 and 23.2\%, respectively); the percentage of current cigarette smokers was slightly greater, and that of ex-smokers slightly less, among men who served in Southeast Asia. However, in this group, a powerful gradient in the cigarette smoking patterns with respect to combat was observed (see Fig. 7). Whereas $39.3 \%$ of low-combat veterans were current cigarette smokers, $45.7 \%$ of medium-combat men and $56.2 \%$ of high-combat men were smokers. This is an important finding. Of the men who served in Southeast Asia, 31.8\% of the low-combat group managed to give up smoking cigarettes prior to this study, compared to $25.5 \%$ of the medium combat group and $21.9 \%$ of those who were in heavy combat.

In addition to cessation, we investigated changes in quantity smoked following military service. Table 7 shows the percentage distribution of these changes (smoking more, less, or the same amount). About half of the current smokers who had not been in Southeast Asia said they had increased their cigarette consumption since leaving the service, while $65.8 \%$ of in-country subjects also said so. But the percentage of smokers who increased their daily consumption was highest among men who experienced heaviest combat: $77.0 \%$ of this group, compared with $57.1 \%$ of the low-combat group.

About one-tenth of all study subjects stated that they do not consume alcohol. There were no significant differences in abstinence between those who served in Southeast Asia and those who served elsewhere, nor between subjects with different levels of combat. There was no overall difference in number of days per

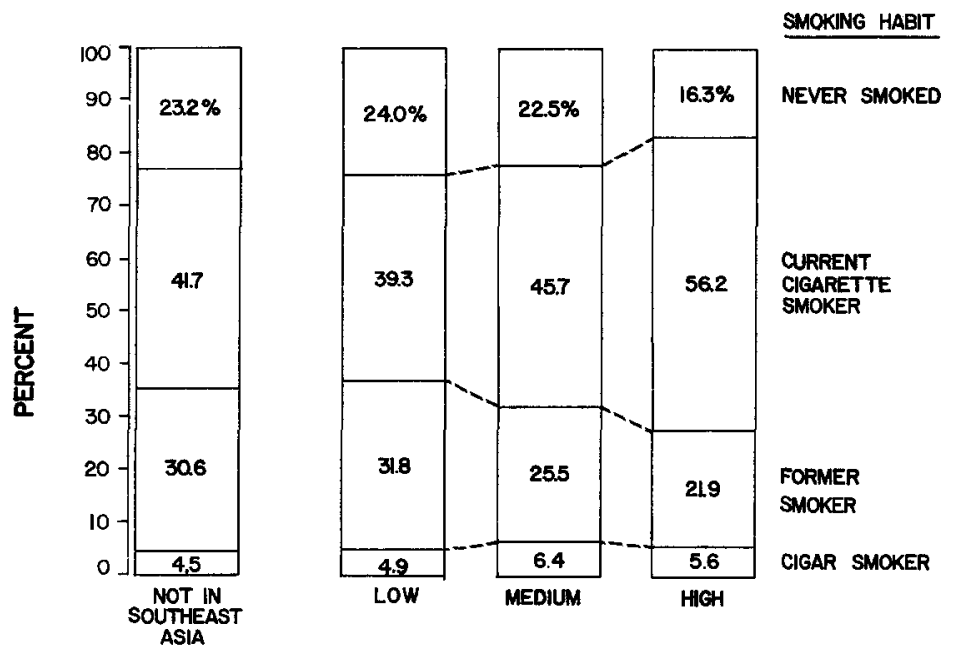

LEVEL OF COMBAT IN SOUTHEAST ASIA

FIG. 7. Distribution of current smoking habits by level of combat in Vietnam. 
TABLE 7

Change in Daily Quantity Smoked by Current Cigarette Smokers According to Service in Southeast Asia and LeVel of Combat

\begin{tabular}{lccccc}
\hline & $\begin{array}{c}\text { Not in } \\
\text { Southeast } \\
\text { Change }\end{array}$ & $\begin{array}{c}\text { All } \\
\text { Asia }\end{array}$ & Southeast & \multicolumn{3}{c}{ Level of combat } \\
\cline { 5 - 6 } & Asia & Low & Medium & High \\
\hline No change & $41.8 \%$ & $28.7 \%$ & $36.2 \%$ & $28.5 \%$ & $17.4 \%$ \\
Smoke more & 51.5 & 65.8 & 57.1 & 67.2 & 77.0 \\
Smoke less & 6.7 & 5.5 & 6.7 & 4.3 & 5.6 \\
Total & 100.0 & 100.0 & 100.0 & 100.0 & 100.0 \\
\hline
\end{tabular}

week that veterans who served in Southeast Asia drank alcohol, compared to other veterans. There was, however, a slight increase in days per week among high-combat veterans, compared to those with low combat (16.5\% drank daily compared to 13.8). The relationship between days per week of drinking and Agent Orange exposure was initially similar to that for combat, but disappeared after adjustment for combat.

We next studied the quantity of alcohol consumed. Since not all men drank alcohol daily, subjects were classified according to the number of days per week they drank. The differences between the mean number of drinks of each alcoholic beverage were investigated by analysis of variance to determine whether they differed according to (a) days per week consumed, (b) service in Southeast Asia vs elsewhere, or (c) level of combat. For three types of beverages, the mean number of drinks differed significantly with days per week; subjects who drank more often tended to consume greater numbers of drinks on those occasions when they drank. For instance, Southeast Asia veterans who drank beer once per week or less consumed on average 3.8 to 4.1 drinks, but those who drank daily consumed on average 6.2 beers $(P<0.001)$. Wine and liquor also were consumed insignificantly greater amounts by veterans who drank more frequently $(P<0.01$ and $P<0.001$, respectively).

The mean number of drinks of beer and of liquor differed significantly between Southeast Asia veterans and controls $(P<0.001$ and $P<0.01$, respectively). These differences disappeared completely after adjustment for level of combat by analysis of covariance. Evidently, the higher levels of beer and liquor consumption in the Southeast Asia subjects could be explained by their exposure to combat. There was no statistical difference between Southeast Asia veterans and controls in amount of wine consumed.

A quantitatively crude but useful estimate of total number of drinks per week was developed by summing the number of drinks per day of beer, wine, and liquor, and multiplying this sum by an approximate number of days per week of alcohol consumption. This calculation assumes that a subject who states he drinks wine and beer, for example, drinks the stated amounts of both beverages on the same days that he drinks. In addition, because the number of days per week was reported in categories rather than as actual number of days, the number of days per week alcohol is drunk was approximated by the following correspondence: if 
a subject stated he drank alcohol less than once per week he was assigned 0.5 days; weekend drinkers were assigned 1.5 days; drinkers 2 to 3 days per week were assigned 2.5 days; 4 to 6 day drinkers were assigned 5 days; and daily drinkers were assigned 7 days.

Table 8 shows the extent to which the veterans consumed alcoholic beverages each week, as computed by this formulation, according to service history and combat. The extent of consumption by veterans who served in Southeast Asia, 18.5 per week, was significantly greater than the 16.0 drinks consumed by other veterans $(P<0.001)$. The number of estimated drinks per week increased with level of combat among Southeast Asia veterans. Regression analysis predicted the number of drinks per week (as calculated by this measure) as a function of combat level with a highly significant coefficient $(P<0.001)$.

More than two-thirds of all study subjects changed their drinking habits in some way since leaving the service. Figure 8 shows the post-service changes in alcohol consumption, according to service in Southeast Asia or elsewhere, and level of combat. Subjects were asked to describe their current habit as "slightly more," "much more," "slightly less," or "much less" than formerly, or else to state there was no change. Veterans who served in Southeast Asia exhibited considerably more variation in their habit than did the other veterans, and were about equally split between increased and decreased drinking. But as Fig. 8 shows, combat level was strongly associated with increased drinking habits: $35.2 \%$ of the high-combat group stated they drank "much more" since leaving the service, compared to $13.0 \%$ of the low-combat group. Conversely, proportionately fewer high-combat veterans reduced their drinking, compared to low-combat veterans or non-Southeast Asia controls.

We also asked, "Have you ever had a serious drinking problem?" Veterans with service in Southeast Asia were more likely to respond affirmatively $(16.1 \%$ vs $11.5 \%$, odds ratio $=1.5, P<0.001$ ). Table 9 shows that the percentage of Southeast Asia veterans who reported a drinking problem increased with level of combat; the difference between low-combat Southeast Asia veterans and controls with no service in Southeast Asia was not significant. The odds ratios in favor of

TABLE 8

Average Weekly Consumption ${ }^{a}$ of all Alcoholic Beverages, According to Service in SOUTHEAST AsIA, AND LeVEl OF COMBAT

\begin{tabular}{lcc}
\hline & $\begin{array}{c}\text { Extent of drinking } \\
\text { each week }\end{array}$ & Number of subjects \\
\hline $\begin{array}{l}\text { Not in Southeast Asia } \\
\text { Southeast Asia }\end{array}$ & 16.0 & 3254 \\
$\quad$ All & 18.5 & 1824 \\
Combat level & & 712 \\
$\quad$ Low & 16.4 & 742 \\
Medium & 18.4 & 370 \\
$\quad$ High & 22.8 & \\
\hline
\end{tabular}

${ }^{a}$ Estimated as drinks per day times days per week that subject consumed alcohol.

${ }^{b}$ Low combat. 

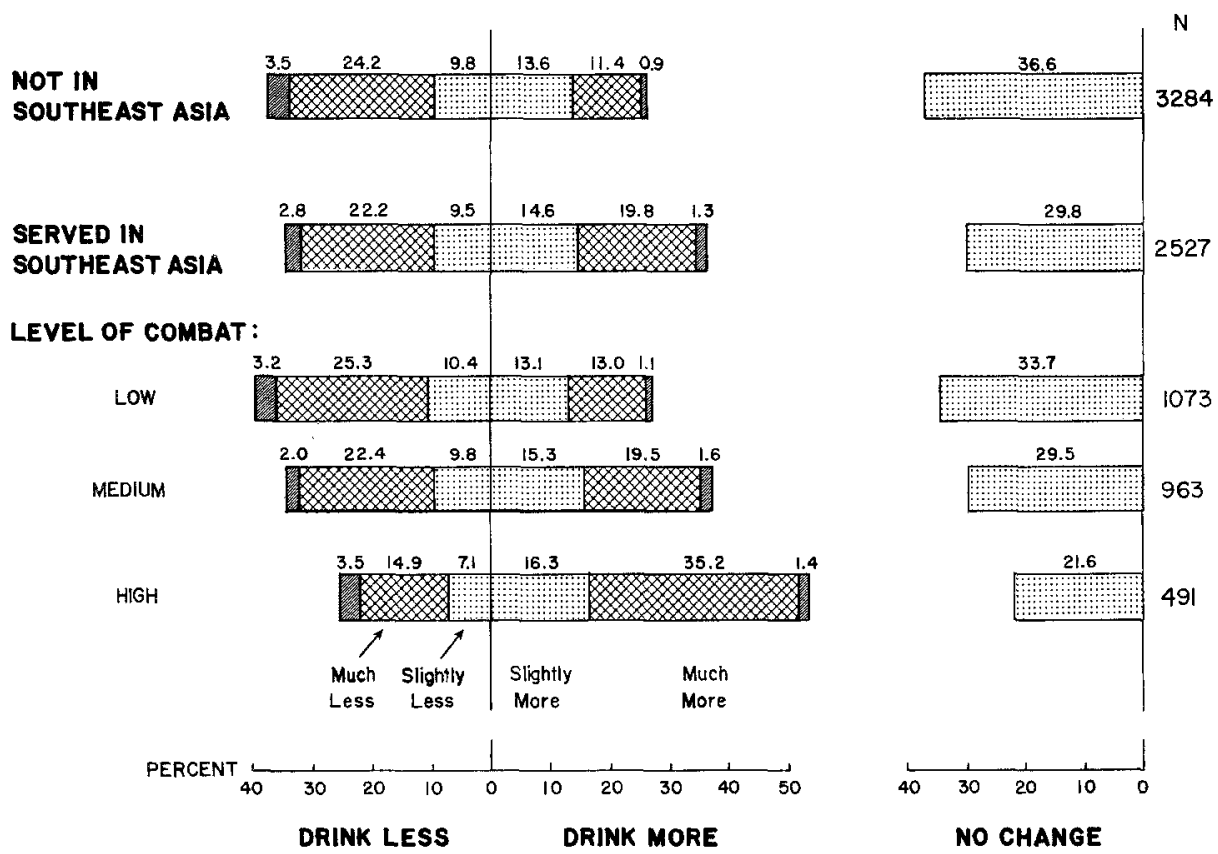

FIG. 8. Distribution of changes in drinking habits following discharge from the military, according to level of combat. Darkest bars indicate more (right side) or less (left side) drinking, but not how much.

giving a positive response to this question were 1.1, 1.6, and 2.2 for low, medium, and high combat, respectively (Mantel test for trend $P<0.001$ ).

Subjects were asked whether they were current users of any of a variety of licit and illicit drugs and medications, and the frequency of such use. There were no differences in frequency of consumption of nonprescription medications (headache remedies, vitamins, food supplements) between men who served in Southeast Asia and those who served elsewhere. Men who had been in Southeast Asia

TABLE 9

Response to “Have You Ever Had a Serious Drinking Problem?", according to Military Service and Level of Combat in Southeast Asia

\begin{tabular}{|c|c|c|c|c|c|}
\hline & \multicolumn{2}{|c|}{ Yes } & \multicolumn{2}{|c|}{ No } & \multirow{2}{*}{$\begin{array}{l}\text { Odds } \\
\text { ratio }\end{array}$} \\
\hline & (No.) & $\%$ & (No.) & $\%$ & \\
\hline Not in Southeast Asia ${ }^{a}$ & 398 & 11.5 & 3054 & 88.5 & 1.0 \\
\hline \multicolumn{6}{|l|}{ Southeast Asia } \\
\hline All Combat level & 406 & 16.1 & 2120 & 83.9 & $1.5^{* * *}$ \\
\hline Low & 133 & 12.4 & 939 & 87.6 & 1.1 \\
\hline Medium & 164 & 17.1 & 796 & 82.9 & 1.6 \\
\hline High & 109 & 22.1 & 385 & 77.9 & $2.2^{* * *}$ \\
\hline
\end{tabular}

${ }^{a}$ Low combat.

Combat level ${ }^{* * *} P<0.001$ for trend. 
were more likely to be regular users of sleeping pills or tranquilizers (6\% vs $3 \%$, $P<.05)$. The two groups did not differ in the percentage of men who admitted regular usage of amphetamines or uppers $(1 \%)$ or marijuana or hashish $(3 \%)$. Six percent had used marijuana occasionally. No subjects admitted to regular use of cocaine or heroin, although about $2 \%$ said they had used cocaine occasionally.

\section{DISCUSSION}

The data presented here graphically illustrate the wide-ranging adverse social and behavioral effects of the combat experience-not simply service experiencein Southeast Asia. The trends are clearly apparent and highly significant in both the social and statistical meaning of that term. Men who faced high levels of combat intensity are at greater risk for divorce and for generally being less happy and satisfied with their lives, their marriages, their role as fathers, and as sexual human beings. Although in this population they have reached comparatively high levels of education, they earn significantly less money than peers of their same age and educational attainment.

The Vietnam veterans who have returned from combat and whom we have studied here exhibit higher levels of behavioral disturbances. Their mean scores for depression, anxiety, irritation, feelings of helplessness, and physical signs of depression are significantly worse than those of their peers who served in Southeast Asia but experienced low levels of combat intensity. And these effects are enduring: for most approximately 15 years have elapsed since their combat experiences. As we shall see in the papers which follow, on post-traumatic stress disorder (PTSD) and on general health effects, these signs of psychological dysfunction are significantly correlated with other serious mental and physical health effects.

Our data show that the lifestyle of combat veterans also places them at greater risk for poor health. They smoke, drink, and take prescription drugs at a significantly higher rate than their noncombat peers, both with and without service in Southeast Asia. They have had, on the whole, a more difficult time in reducing their smoking and drinking habits than the other men in the study.

The observed change in smoking habits is also an important finding since cessation of smoking is one of the most effective ways of reducing of risk for a number of illnesses, but it is also a very difficult process for many people. This is consistent with theories of smoking maintenance as a stress-relieving action.

It is also important to discuss the generalizability of these data. Unlike the veteran population at large, The American Legion sample is highly homogeneous. It is representative of the solid "middle section" of white America, with its relatively high educational and income level and marriage rate. It is also a sample that has been resocialized sufficiently to have joined The American Legion, which the majority view as a social organization (see Paper 1). This study is an American Legion-sponsored study of a random sample of its Vietnam Era members. The socioeconomic and demographic characteristics of Legionnaires lead us to conclude that the data here represent a "best case" analysis of the potential effects of the war experience on many other veterans. We suggest that those with less social support and fewer economic supports will be suffering more, not less. 
We suggest that the homogeneity of the sample strengthens the study in that the absence of potential serious confounding factors, such as race and chronic unemployment, has simplified the analysis and allowed us to focus on the effects of the war experience itself. In addition, there is an overrepresentation, by design, of men with combat zone experience, enabling us to focus more closely on the effects of Vietnam combat.

Internal consistency of the findings also lends credibility to the study. These analyses compared peer groups with and without service in Southeast Asia, and throughout, consistent results were obtained. The data are also consistent with the work of other researchers, discussed earlier, who have studied the effects of the combat experience on Vietnam Veterans (e.g., Egendorf et al., 1981; Figley, 1978; Card, 1980).

We also note that our data give some indication that certain behavioral effects related to combat intensity may be exacerbated by concurrent exposure to herbicides and indicate the need for further exploration of this issue. We observed that while herbicide exposure could not independently predict the psychosocial outcomes, it did significantly predict the outcomes when entered as a crossproduct term with combat. Such an observation could be explained by a model in which herbicide exposure is significantly related to the outcome at high levels of combat. Such a finding is consistent with experimental and epidemiological data which demonstrate the increased vulnerability of organisms to toxic exposures when simultaneously exposed to known stressors (House et al., 1979).

As we have discussed in the preceding paper, the confounding effect of combat is very powerful. When we control for combat, we lose statistical significance of the independent herbicide effects observed. But it is noteworthy that anxiety and physical signs of depression were the psychological effects for which Agent Orange exposure, measured by the $\mathrm{OE} 3$ index, was a significant predictor. These symptoms are significantly correlated with the health conditions which we have also found to be related to the OE3 index and which are described in the following paper in this series (Stellman et al., 1988b). Further study, with a larger population, is necessary in order to control the confounding effects of combat more effectively and thereby estimate more accurately the effects of herbicides on psychosocial well-being.

\section{REFERENCES}

Bogen, G. (1979). Symptoms in Vietnam veterans exposed to Agent Orange (letter). J. Amer. Med. Assoc. 242, 2391.

Caplan, R. D., Cobb, S., French, J. R. P., Jr., Van Harrison, R., and Pinneau, S. R., Jr. (1975). “Job Demands and Worker Health: Main Effects and Occupational Differences.' U.S. DHEW Publication No. (NIOSH) 75-160, Washington, DC.

Card, J. (1980). "Lives After Vietnam: The Personal Impact of Military Service." Lexington Books, Lexington, MA.

Dohrenwend, B. P., Shrout, P. E., Egri, G., and Mendelsohn, S. S. (1980). Nonspecific psychological distress and other dimensions of psychopathology: Measures for use in the general population. Arch. Gen. Psychol. 37, 1229-1235.

Egendorf, A., Kadushin, C., Laufer, R. S., Rothbart, G., and Sloan, L. (1981). "Legacies of Vietnam: Comparative Adjustment of Veterans and their Peers." U.S. Gov. Printing Office, Washington, DC. 
Figley, C. R. (1978). Psychosocial adjustment among Vietnam veterans. In "Stress Disorders among Vietnam Veterans"' (C. R. Figley, Ed.). Brunner/Mazel, New York.

Frye, J. S., and Stockton, R. A. (1982). Discriminant analysis of posttraumatic stress disorder among a group of Vietnam veterans. Amer. J. Psychiatry 139, 52-56.

House, S. J., Wells, J. A., Landerman, L. R., McMichael, A. J., and Kaplan, B. H. (1979). Occupational stress and health among factory workers. Health Soc Behav 20, 139-196.

Poland, A., Smith, D., Metter, G., and Possick, P. (1971). A health survey of workers in a 2,4-D and 2,4,5-T plant. Arch. Environ. Health 22, 316-327.

Quinn, R., and Shepard, L. J. (1974). "The 1972-73 Quality of Employment Survey." University of Michigan Survey Research Center, Ann Arbor.

Roberts, W. R., Penk, E., Gearing, M. L., Robinowitz, R., Dolan, M. P., and Patterson, C. T. (1982). Interpersonal problems of Vietnam combat veterans with symptoms of posttraumatic stress disorder. J. Abnorm. Psychol. 91, 444-450.

Snow, B. R., Stellman, J. M., Stellman, S. D., Sommer, J. F., Jr. (1988). Post-traumatic stress disorder among American Legionnaires in relation to combat experience in Vietnam: Associated and contributing factors. Environ. Res. 47, 175-192.

Stellman, S. D., and Stellman, J. M. (1986). Estimation of exposure to Agent Orange and other defoliants among American troops in Vietnam: A methodological approach. Amer. J. Ind. Med. 9, 305-321.

Stellman, S. D., Stellman, J. M., and Sommer, J. F., Jr. (1988a). Combat and herbicide exposures in Vietnam among a sample of American Legionnaires. Environ. Res. 47, 112-128.

Stellman, S. D., Stellman, J. M., and Sommer, J. F., Jr. (1988b). Health and reproductive outcomes among American Legionnaires in relation to combat and herbicide exposure in Vietnam. Environ. Res. 47, 150-174.

Stellman, J. M., and Snow, B. R. (1986). Occupational safety and health hazards and the psychosocial health and well-being of workers. In "Health and Industry: A Behavioral Medicine Perspective" (M. F. Cataldo and T. J. Coates, Eds.). Wiley, New York.

Suskind, R. R., and Hertzberg, V. S. (1984). Human health effects of 2,4,5-T and its toxic contaminants. J. Amer. Med. Assoc. 251, 2372-2380.

Worthington, E. R. (1977). Post-service adjustment and Vietnam era veterans. Mil. Med. 142, 865866.

Worthington, E. R. (1978). Demographic and pre-service variables as predictors of post-military service adjustment. In "Stress Disorders among Vietnam Veterans"' (C. R. Figley, Ed.). Brunner/ Mazel, New York. 